\title{
Effects of vitrification and post-thawing interval on the cytoskeleton and subsequent fertilization rate of in vitro derived bovine oocytes
}

\author{
T.L. Nedambale ${ }^{1,2,3 \#}$, F. Du ${ }^{2}$, J. Xu ${ }^{2}$, X.C. Tian ${ }^{1}$ and X. Yang ${ }^{1}$ \\ ${ }^{1}$ University of Connecticut, Centre for Regenerative Biology/Department of Animal Science, \\ 1392 Storrs Rd., U-4243, Storrs, CT, 06269-USA \\ ${ }^{2}$ Evergen Biotechnologies, Inc., Storrs, CT, 06269-USA \\ ${ }^{3}$ ARC-LBD: Animal Production Institute, Indigenous Genotype Physiology and Biotechnology Development, \\ Private Bag X2, Irene 0062, South Africa
}

\begin{abstract}
Vitrification may alter the cytoskeleton (microtubule, meiotic spindle, microfilament, etc.) and the subsequent fertilization rate of in vitro derived bovine oocytes. This study was conducted to evaluate the effect of vitrification and post-thawing incubation periods on the cytoskeleton and fertilization rate of in vitro matured (IVM) bovine oocytes. Following 22 h of IVM, 184 fresh matured oocytes (MO) were immediately fertilized in vitro and served as a control. The remaining MO (1009) were then vitrified by the solid surface vitrification method. Immediately after thawing, $\mathrm{MO}$ were incubated in maturation medium in $5 \% \mathrm{CO}_{2}$ at $39{ }^{\circ} \mathrm{C}$ for 0 , 30, 60, 90 and 120 min respectively. Following incubation, half of the MO from each vitrifiedthawed treatment group (0, 30, 60, 90, and $120 \mathrm{~min}$ ) was stained with fluorescein isothiocyanate conjugated (FITC) and propidium iodide (PI) to evaluate the microtubule and DNA or spindle under laser-scanning confocal microscopy. The remaining half from the vitrified-thawed MO treatment groups was washed three times in Brackett and Oliphant's fertilization medium and in vitro fertilized. Cleavage and blastocyst rates were recorded $48 \mathrm{~h}$ post-fertilization. Results demonstrated that vitrification damaged MO zona pellucida (ZP), microtubule (MT), meiotic spindle (MS), and caused chromosomal fragmentation. Both the cleavage (84\%) and blastocyst rates (50\%) of the control group were significantly higher compared to the vitrifiedthawed treatment groups. However, extending the incubation period of vitrified MO to 120 min after thawing (prior to fertilization) improved cleavage (65\%) and blastocyst (13\%) rates $48 \mathrm{~h}$ post-fertilization. Fertilizing vitrified MO immediately (0 min group) after thawing resulted in the lowest cleavage (42\%) and blastocyst (1.9\%) rates. In conclusion, vitrification reduces the subsequent fertilization rate of MO, however, a prolonged post-thawing incubation period (120 min) improves survival, cleavage and blastocyst formation rates, and enhances the reorganization of MO’s cytoskeleton (MT and MS).
\end{abstract}

Keywords: Vitrification, cytoskeleton, bovine, oocytes, fertilization, in vitro matured

\#Corresponding author. E-mail: lucky@arc.agric.za, nedambale@hotmail.com

\section{Introduction}

In the mid 80's, vitrification entered the mainstream of animal embryo technologies (Rall \& Fahy, 1985) as an alternative cryopreservation technique to the traditional slow-freezing method. Since then vitrification of mammalian embryos and oocytes has been the subject of many investigations, and has been achieved simply by plunging the sample directly into liquid nitrogen (Rall \& Fahy, 1985; Arav et al., 2002; Nedambale et al., 2006). Furthermore, several attempts to improve the survival rate of oocytes pioneered the establishment of different cryopreservation techniques such as the solid surface vitrification (SSV) method (Dinnyés et al., 2000), open-pulled-straw (OPS, Vajta et al., 1998) and Cryo-loop (Lane \& Gardner, 2001). Despite this extensive research over the past 20 years, limited progress has been made. The survival rate of cryopreserved and thawed oocytes in terms of development to the blastocyst stage remains low, and only few calves have been born (Vajta et al., 1998). Perhaps vitrification of oocytes is currently one of the greatest challenges facing female gamete storage and assisted reproductive technologies (ART). Effective means of cryopreservation of bovine oocytes followed by the higher survival rate would be important for improving blastocyst formation rate following IVF. Thus, more research is needed in order to gain a better understanding of the morphological cytoskeleton of bovine oocytes that could be crucial in providing a cryobiological basis to develop a more suitable vitrification protocol. The present study was carried out to 
evaluate the effects of vitrification and post-thawing intervals on the cytoskeleton and subsequent fertilization rate of in vitro derived bovine oocytes.

\section{Materials and Methods}

All chemicals used in this study were purchased from Sigma Chemical Co. (St. Louis, MO-USA), unless otherwise indicated. Bovine oocytes were aspirated from ovaries collected at an abattoir (Yankton, SD, USA), and then matured in Medium199 plus 10\% fetal bovine serum (FBS, Hyclone, Logan, UT, USA), 1\% antibiotic/antimycotic (Gibco, Grand Island, NY, USA) and $10 \mathrm{ng} / \mathrm{mL}$ epidermal growth factor (EGF) in 5\% $\mathrm{CO}_{2}$ in air at $39{ }^{\circ} \mathrm{C}$ for $22 \mathrm{~h}$ (Nedambale et al., 2004). Following $22 \mathrm{~h}$ of in vitro maturation (IVM), 184 matured oocytes (MO; fresh) served as a control and were immediately fertilized. The remaining MO (1009) were randomly aliquoted into five groups: $0 \mathrm{~min}(\mathrm{n}=199), 30 \mathrm{~min}(\mathrm{n}=201), 60 \mathrm{~min}(\mathrm{n}=202), 90 \mathrm{~min}$ $(n=204)$, and $120 \min (n=203)$. The MO within their respective groups were then vitrified by a solid surface vitrification method (35\% (v/v) EGF + $0.4 \mathrm{M}$ trehalose + 5\% (w/v) PVP in M199 + 20\% FBS (Dinnyés et al., 2000). Immediately after thawing, $\mathrm{MO}$ were incubated in a maturation medium in $5 \% \mathrm{CO}_{2}$ at $39{ }^{\circ} \mathrm{C}$ for 0 , 30, 60, 90 and 120 min, respectively. Following incubation, half of the MO from each vitrifiedthawed treatment groups $(0,30,60,90$, and $120 \mathrm{~min})$ was stained with fluorescein isothiocyanate conjugated (FITC) and Propidium iodide (PI) to visualize microtubule and DNA or spindle under laser-scanning confocal microscopy (Chang et al., 2004). However, only 20 oocytes $(n=20)$ per treatment group were examined. The remaining half of the vitrified-thawed MO group was washed three times in Brackett and Oliphant's fertilization medium as described previously (Nedambale et al., 2004), and then transferred into $50 \mu \mathrm{L}$ drops in a Petri dish covered with mineral oil (Becton Dickinson, Franklin Lakes, NJ, USA). The dishes were incubated at $39{ }^{\circ} \mathrm{C}$ in $5 \% \mathrm{CO}_{2}$ in the air. A semen straw (ABS, DeForest, WI, USA) was thawed at $38{ }^{\circ} \mathrm{C}$ for $1 \mathrm{~min}$, and washed two times by centrifugation at $453 \mathrm{x} \mathrm{g}$ for $8 \mathrm{~min}$. Following the final wash, sperm motility, and concentration were determined. Subsequently, sperm pellets were re-suspended into a volume of $250 \mu \mathrm{L}$ per treatment group. Forty micro-litres $(40 \mu \mathrm{L})$ of the sperm were added to each fertilization drop, giving a total concentration of $1 \times 10^{7}$ spermatozoa/mL. Oocytes were then incubated with the sperm for $6 \mathrm{~h}$ in $5 \% \mathrm{CO}_{2}$ in air at $39{ }^{\circ} \mathrm{C}$. After $6 \mathrm{~h}$ of IVF, oocytes were washed six times in TL-HEPES (Bio-Whittaker, Walkersville, MD, USA) and cultured in our previously described sequential KSOM-SOF culture system (Nedambale et al., 2004). The day of fertilization is designated as day zero. Embryo cleavage rate was recorded $48 \mathrm{~h}$ post-fertilization and total blastocyst rate on Day 8.

The data was subjected to a one-way analysis of variance (ANOVA) following arc sine transformation (data replicated 4 times). Differences between post-thawing interval groups were determined using Bonferroni's test for pair-wise comparison of means, $\mathrm{P}<0.05$ was considered significant.

\section{Results and Discussion}

The results of this study demonstrate that fertilizing vitrified matured oocytes immediately ( 0 min group) after thawing resulted in the lowest $(\mathrm{P}<0.05)$ cleavage $(42 \%)$ and blastocyst $(1.9 \%)$ rates. However, extending the incubation period of vitrified MO to 120 min after thawing improved $(\mathrm{P}<0.05)$ cleavage (65\%) and blastocyst (13\%) rates post-fertilization. There were no statistical differences in the cleavage and blastocyst formation rates of vitrified and thawed oocytes, which were derived from 30 (46 and 9\%), 60 (45 and $11 \%$ ), and 90 (50 and 12\%) min groups, respectively. As expected, cleavage (84\%) and blastocyst rates (50\%) of fresh MO (control) were significantly higher compared to the vitrified-thawed groups. Significantly higher survival rates were recorded in the 0 (82\%) and 30 (80\%) min groups than in the 60 (61\%), 90 (66\%) and 120 (67\%) min groups, respectively (Figure 1).

As shown in Figure 2, in a retrospective comparison among the treatment groups following FITC and PI staining, a significantly higher MO in 0 min group had abnormal spindle (50\%) and microtubule (35\%), compared to the other groups. However, microtubule (22 and 15\%) and spindle (13 and 9\%) of vitrified MO reorganized and showed less damages following incubation for longer periods (90, and $120 \mathrm{~min}$, respectively) post-thawing. The highest overall damages (OA) in vitrified-thawed MO were observed in 0 (92\%) and 30 (69\%) min compared to the other groups. 


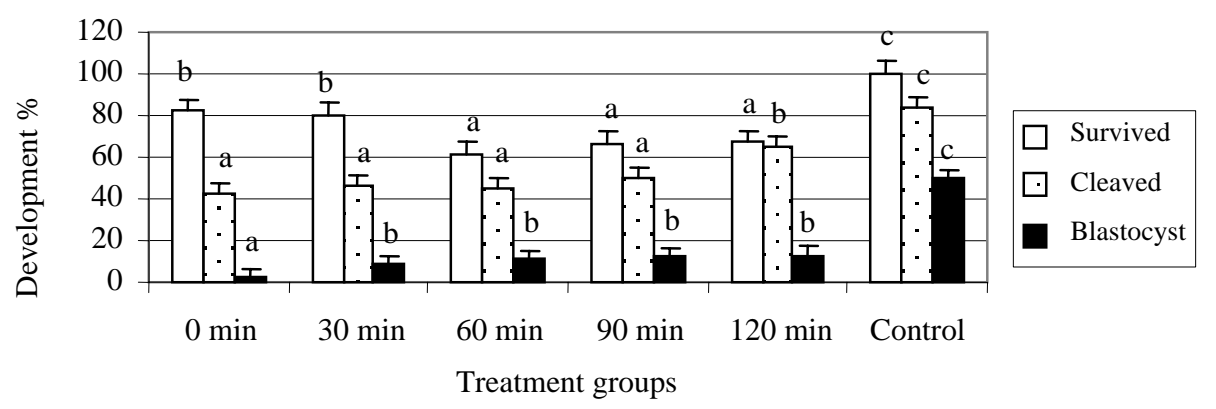

Figure 1 Post-thawing survival rate and embryo development following in vitro fertilization of fresh (control) and vitrified bovine oocytes fertilized at different intervals. ${ }^{\mathrm{a}, \mathrm{b}, \mathrm{c}}$ Values with different superscripts across the bars are significantly different $(\mathrm{P}<0.05)$
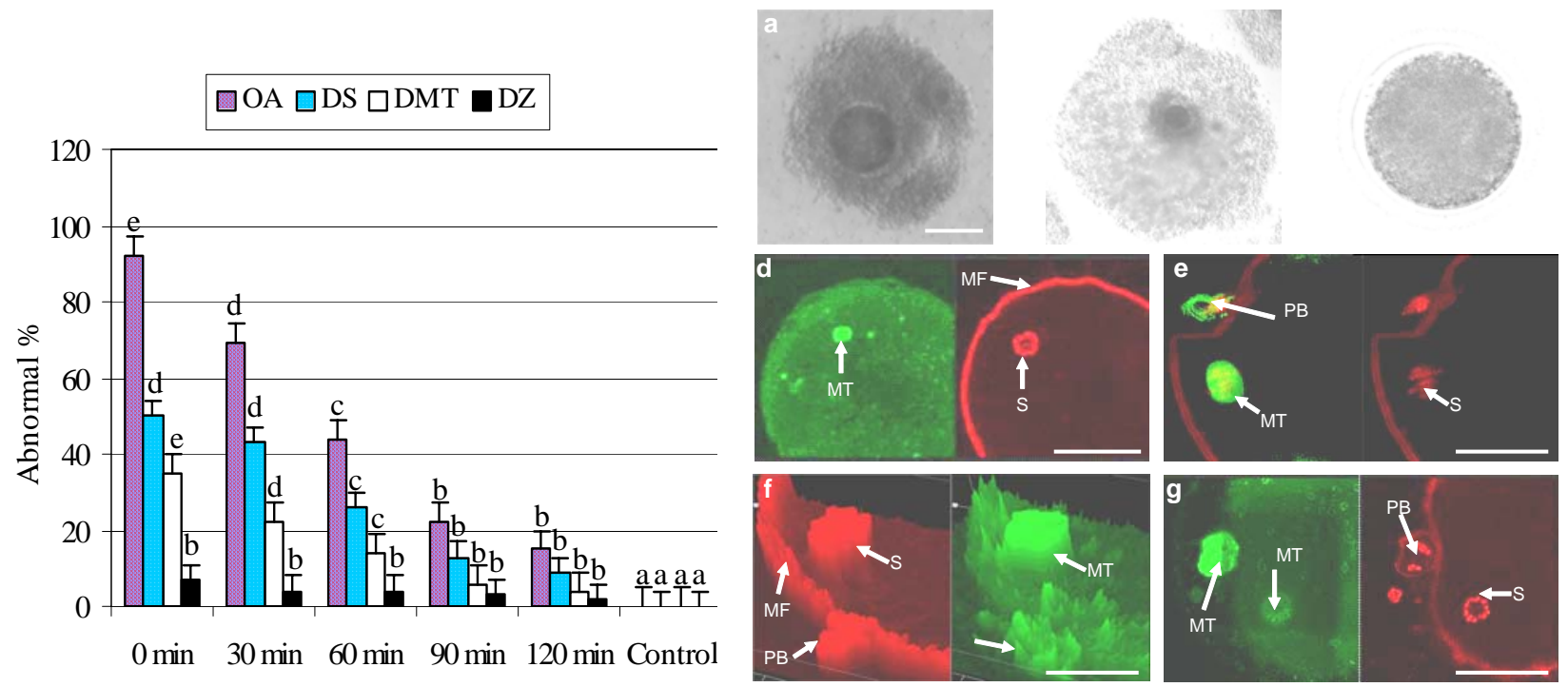

Figure 2 Morphological evaluation of cytoskeleton structure (Microtubule (MT), microfilament (MF) and meiotic spindle (S) of vitrified-thawed bovine oocytes. The histogram illustrates all the abnormalities observed at different groups. ${ }^{\mathrm{a}-\mathrm{e}}$ Values with different superscripts across bars are significantly different $(\mathrm{P}<0.05)$. The OA $=$ overall abnormalities, DS = damaged spindle, DMT = damaged microtubule, DZ = damaged zona. (a) Normal immature bovine oocyte after ovary aspiration. (b) Matured oocyte with well-expanded cumulus cells selected for vitrification $22 \mathrm{~h}$ after IVM. (c) Denuded matured oocyte following removal of cumulus cells from control group (not vitrified). (d) Normal MT attached to S and MF circling the cytoplasm of the oocyte, control group (not vitrified). (e) A normal MT attached to abnormal or damaged S, and abnormal MT attached to damaged small polar body (PB), derived from 0 min group. (f) Three dimension (3-D) structure taken from different level positions that combined to form a well-defined 3-D structure of a normal spindle, MF, and PB. Subsequently, few or depolymerised MT attached to PB and normal MT attached to PB, from the 120 min group. (g) Normal MT attached to normal PB, and abnormal or small MT attached to normal spindle of matured oocyte 120 min post-thawing. Bar $=100 \mu \mathrm{m}$

This study demonstrated that vitrification adversely affects subsequent in vitro fertilization and survival rates and the ability to reorganize the damaged meiotic spindle and microtubule of MO (Figure 2). However, these adverse effects tended to improve significantly by post-thawing incubation prior to in vitro fertilization. This seems to allow reorganization of the MO's cytoskeleton and to be time dependent. These results are consistent with the findings of Pickering et al. (1990), who reported that exposure of human or mouse oocytes to cryopreservation procedures and low temperature disrupts its cytoskeleton. Zona pellucida 
has also been found to be damaged and hardened due to cryopreservation resulting in decreased fertilization rates (Fuku et al., 1995).

\section{Conclusion}

In conclusion, vitrification reduced the subsequent survival and fertilization rates of in vitro matured oocytes. However, extended post-thawing incubation periods $(120 \mathrm{~min})$ improved cleavage and blastocyst formation rates post-fertilisation. Microtubule and spindle reorganization of vitrified-thawed oocytes incubated for 120 min prior to fertilization showed better reorganization and less damage than all other groups of vitrified oocytes considered in this study

\section{Acknowledgement}

This study was supported financially by a joint grant, USDA and the National Research Foundation (NRF) of South Africa.

\section{References}

Arav, A., Yavin, S., Zeron, Y., Natan, D., Dekel, I. \& Gacitua, H., 2002. New trends in gamete's cryopreservation. Mol. Cell Endocrinol. 187, 77-81.

Chang, C.C., Nagy, Z.P., Abdelmassih, R., Yang, X. \& Tian, X.C., 2004. Nuclear and microtubule dynamics of $\mathrm{G}_{2} / \mathrm{M}$ somatic nuclei during haploidization in germinal vesicle-stage mouse oocytes. Biol. Reprod. 70, 752-758.

Dinnyés, A., Dai, Y., Jiang, S. \& Yang, X., 2000. High developmental rates of vitrified bovine oocytes following parthenogenetic activation, in vitro fertilization, and somatic cell nuclear transfer. Biol. Reprod. 63, 513-518.

Fuku, E.J., Liu, J. \& Downey, B.R., 1995. In vitro viability and ultrastructural changes in bovine oocytes treated with a vitrification solution. Mol. Reprod. Dev. 40, 177-185.

Nedambale, T.L., Dinnyés, A., Groen, W., Dobrinsky, J.R., Tian, X.C. \& Yang, X., 2004. Comparison on in vitro fertilized bovine embryos cultured in KSOM or SOF and cryopreserved by slow freezing or vitrification. Theriogenology 62, 437-449.

Nedambale, T.L., Du, F., Yang, X. \& Tian, X.C., 2006. Higher survival rate of vitrified and thawed in vitro produced bovine blastocysts following culture in defined medium supplemented with [beta]-mercaptoethanol. Anim. Reprod. Sci. 93, 61-75.

Lane, M. \& Gardner, D.K., 2001. Vitrification of mouse oocytes using a nylon loop. Mol. Reprod. Dev. 58, 342-347.

Pickering, S.J., Braude, P.R., Johnson, M.H., Cant, A. \& Currie, J., 1990. Transient cooling to room temperature can cause irreversible disruption of the meiotic spindle in the human oocyte. Fertil. Steril. 54, 102-108.

Rall, W.F. \& Fahy, G.M., 1985. Ice-free cryopreservation of mouse embryos at -196 degrees C by vitrification. Nature 313, 573-575.

Vajta, G., Holm, P., Kuwayama, M., Booth, P.J., Jacobsen, H., Greve, T. \& Callesen, H., 1998. Open Pulled Straw (OPS) vitrification: A new way to reduce cryoinjuries of bovine ova and embryos. Mol. Reprod. Dev. 51, 53-58. 